\title{
A Comparative Study of TOPSIS Method via Different Distance Measure
}

\author{
Pranjal Talukdar ${ }^{1}$, Palash Dutta ${ }^{2}$ \\ ${ }^{1,2}$ Department of Mathematics, Dibrugarh University, Dibrugarh, Assam, India, 786004 \\ Email: pranjaltalukdar70@gmail.com ${ }^{1}$, palsh.dtt@gmail.com ${ }^{2}$
}

\begin{abstract}
One of the techniques used to support strong decision making in domains where selection of best alternative is highly complex is the Fuzzy TOPSIS method. In TOPSIS procedure different distance measures are used to calculate the distance of each fuzzy number from both Fuzzy Positive Ideal Solution (FPIS) and Fuzzy negative Ideal Solution (FNIS). In this paper, six well known distance measures have been taken into account which are available in literature and applied to TOPSIS procedure to perform a comparative study throughout numerical problems. The main purpose is to compare the stability and reliability of TOPSIS method in decision science through different distance measures. Fuzzy triangular number is used in our entire case study.
\end{abstract}

Keywords- Multicriteria decision making, fuzzy TOPSIS, FPIS, FNIS, Triangular Fuzzy Number, Fuzzy Distance Measure.

\section{INTRODUCTION:}

In many of real world situations, usually decision makers are confronted with multiple criteria to be considered before any decision can be made. The main aim of Multi Criteria Decision Making (MCDM) is to find the best alternative among a set of available alternatives in the presence of various conflicting criteria. In Decision sciences one method which has a great importance in the field of research is Technique for Order Performance by Similarity to Ideal Solution (TOPSIS). In TOPSIS method crisp data are used and further it is extended under fuzzy environment. Crisp data are not always adequate to model in many real life situations where as fuzzy set theory is more suitable to handle such type of situation. For representation of fuzziness in the decision data and group decision making process, linguistic variables are used to access the weight of all criteria and ratings of each alternative with respect to each criterion. TOPSIS views situation with $m$ alternatives as a geometric system with $m$ points in the $n$-dimensional space. It was developed by Hwang and Yoon [1]. TOPSIS defines an index called relative closeness of the positive-ideal solution and the remoteness from the negative-ideal solution which is based upon the concept that the chosen alternative should have the shortest distance from the positive ideal solution (PIS) and farthest from the negative ideal solution (NIS), i.e., the solution that maximizes the cost criteria and minimizes the benefit criteria. The higher value of closeness coefficient indicates that an alternative is closer to FPIS and farther from FNIS simultaneously, i.e., the optimal alternatives is the one which has the shortest distance from the positive ideal solution and the farthest distance from the negative ideal solution. To calculate the closeness coefficient it is necessary to calculate the distance measure between two fuzzy numbers. Many distance measures are available in literature. In computational procedure many problems arises due to the vagueness of ill-defined data.
Several computational procedures for finding the distance between fuzzy numbers have been developed by L. Tran \& L. Duckstein [2], C.H. Cheng [3], W. Voxman [4], P. Diamond [5] and C. Chakraborty et al., [6] and have spread their applicability in different field. The concept of fuzzy set theory is introduced by L. A. Zadeh [7]. Bellman and Zadeh [8] and Zimmermann [9] took the fuzzy sets to deal with problems associated with vague, imprecise and ill concept into standard multicriteria decision making (MCDM) techniques and also proposed that the constrains related to a MCDM problem could be defined as fuzzy sets in the space of alternatives. Baas and Kwakernaak [10] works on classical MCDM methods. Yager [11] illustrated that the best alternative should have the highest membership value. Chen and Hwang [12] presented Multiple person MADM problems with imprecise information is represented in fuzzy terms. Kickert [13] also worked on MADM using fuzzy sets. Dubois and Prade [14], Zimmermann [15], and Ribeiro,[16] divided the MADM methods into two branches. One branch deals with the measurement of performance rating with respect to all attributes of each alternative and the aggregate rating is measured to indicating the global performance of each alternative the other branch deals with ranking of alternatives by ordering the existing alternatives according to the resulted aggregated performance. The technique for order preference by similarity to ideal solution (TOPSIS) is a MCDM method which was originally developed by Hwang and Yoon [1] which are further modified by Yoon in 1987. Liang [17] presented a fuzzy multicriteria decision making based on the concept of ideal and anti-ideal points. Lai et al. [18] developed the proposed TOPSIS for MODM algorithm for solving multiple objective decision making problems .Yoon and Hwang [19], proposed the advanced procedures for TOPSIS methods. Triantaphyllou and Lin [20] developed a fuzzy version of TOPSIS method based on fuzzy 


\section{International Journal of Research in Advent Technology, Vol.7, No.5, May 2019 \\ E-ISSN: 2321-9637 \\ Available online at www.ijrat.org}

arithmetic operation which leads to a fuzzy relative closeness for each alternative. Chen [21] proposed that the rating of each alternative and weight of each criterion which are described by linguistic terms can be expressed in triangular fuzzy number. Then a vertex method is proposed to calculate the distance the two triangular fuzzy numbers this concept are used in TOPSIS method. S. Saghafian, S. R. Hejazi [22], defined a new distance measure and used it to modified TOPSIS method. Abo-Sinna and Enien [23] extend the technique for order preference by similarity ideal solution (TOPSIS) for solving large scale multiple objective programming problems involving fuzzy parameters. Wang and Elhag [24] presents a non-linear programming solution procedure using fuzzy TOPSIS method based on alpha level set. Jahanshahloo et al., [25] study the cases in which determining precisely the exact value of the attributes is difficult, and as a result of this, the attribute values should be considered as intervals. They aim to extend the TOPSIS method for decision making problems with interval data. By extension of the TOPSIS method, they present an algorithm to determine the most preferable choice among all possible choices, when data are interval. Ashtiani, Haghighirad, Makui and Montazer [26] presented an interval-valued fuzzy TOPSIS for solving MCDA problems. Chen and Lee [27] presented an interval type-2fuzzy TOPSIS method to handle fuzzy multiple-attribute group decision-making problems based on interval type-2 fuzzy sets. Yang and Hung [28] utilized TOPSIS for solving a plant layout design problem. In particular, Wang and Lee [29] proposed two operator Up and Lo to find positive-ideal and negative-ideal solutions and used these operators to solve fuzzy multiple-criteria group decision making problem. Chen and Tsao [30] extended the concept of TOPSIS to develop a method for solving MADM problems with interval-valued fuzzy data and compared the results using different distance measures, including Hamming distance, Euclidean distance and their normalized forms. Tran and Duckstein [2] introduced a distance concept based on the interval numbers where the fuzzy number has transformed into an interval number on the basis of the $\alpha$-cut. Sun [31] developed an evaluation model based on the fuzzy analytic hierarchy process (AHP) and fuzzy TOPSIS to help the industrial practitioners for the performance evaluations in fuzzy environment. Brans and Vincke [32], El Hawary [33], Chang and Yeh [34], Kahraman et al., [35] use the interval numbers for ranking alternatives.

In this paper various existing distance measure for triangular fuzzy numbers from literature have taken and a comparison study has been done using TOPSIS method in order to having the proper ranking of the alternatives with respect to different criteria. The main aim of this paper is to determine the stability and reliability of the TOPSIS procedure through different distance measure. Considering the fuzziness in decision data and group decision making process, linguistic variables are used to access the weight of all criteria and the ratings of each alternative with respect to each criterion. The results which are obtained by performing the calculation using different distance measures show that the order ranking of various distance measures is identical, which is more enough to implement the TOPSIS as an effective aid in decision science.

\section{INTRODUCTION TO FUZZY SET THEORY:}

Definition 1 [7] Let $X$ be a space of points. A fuzzy set $A$ in a in $X$ characterized by a membership function $\mu_{A}(x)$ which associates with each point in $X$ a real number in the interval $[0,1]$ with the value of $\mu_{A}(x)$ at $x$ representing the grade of membership of $x$ in $A$.

Definition 2 [36] The height of a fuzzy set is the largest membership grade attained by any element in that set. A fuzzy set $A$ in the universe of discourse $X$ is called the normalized when the height of $\mathrm{A}$ is equal to one.

Definition 3 [36] A fuzzy set $A$ in a universe of discourse $\mathrm{X}$ is convex if and only if $\mu_{A}\left(\lambda x_{1}+\left(1-\lambda x_{2}\right)\right) \geq \min \left(\mu_{A}\left(x_{1}\right), \mu_{A}\left(x_{2}\right)\right)$ for $\quad$ all $x_{1}, x_{2}$ in $\mathrm{X}$ and all $\lambda \in[0,1]$ where min denotes the minimum operator.

Definition 4 [37] A fuzzy number $\tilde{n}$ is a fuzzy subset in the universe of discourse $X$ that is both normal and convex. Fig 1 shows a fuzzy number $\widetilde{n}$.

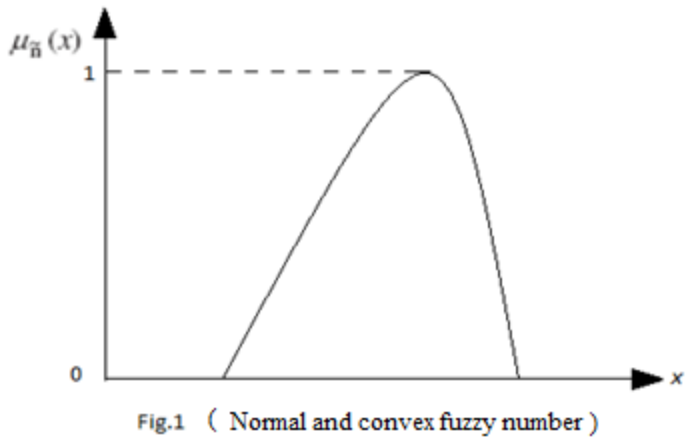

Definition 5 [37] The $\alpha$-cut of a fuzzy number $\tilde{n}$ is defined as:

$\tilde{n}^{\alpha}=\left\{x_{i}: \mu_{\tilde{n}}\left(x_{i}\right) \geq \alpha, x_{i} \in X\right\} \quad$ Where $\alpha \in[0,1] . \tilde{n}^{\alpha}$, which is defined above is a non empty closed bounded interval contained in $\mathrm{X}$ and it can be denoted by $\tilde{n}^{\alpha}=\left[\tilde{n}_{l}^{\alpha}, \tilde{n}_{u}^{\alpha}\right]$ where $\tilde{n}_{l}^{\alpha}$ and $\tilde{n}_{u}^{\alpha}$ are the lower and upper bounds of the closed interval respectively (H.J. Zimmermann, 1991). For a fuzzy number $\widetilde{n}$ if $\widetilde{n_{l}^{\alpha}}>$ 0 and $n_{u}^{\alpha} \leq 1$ for all $\alpha \in[0,1]$ then $\tilde{n}$ is called a standard (normalized) positive fuzzy number.

Definition 6 [38] A triangular fuzzy number $\tilde{n}$ can be defined by a triplet $\left(n_{1}, n_{2}, n_{3}\right)$ The membership function $\mu_{\tilde{n}}(x)$ is defined as $\mu_{\tilde{n}}=\left\{\begin{array}{cc}\frac{x-n_{1}}{n_{2}-n_{1}}, & n_{1} \leq x \leq n_{2} \\ \frac{x-n_{3}}{n_{2}-n_{3}}, & n_{2} \leq x \leq n_{3} \\ 0 & \text { otherwise }\end{array}\right.$.

The fuzzy number $\widetilde{n}=\left(n_{1}, n_{2}, n_{3}\right)$ with $n_{1} \leq n_{2} \leq n_{3}$ is shown in the following figure- 2 . 


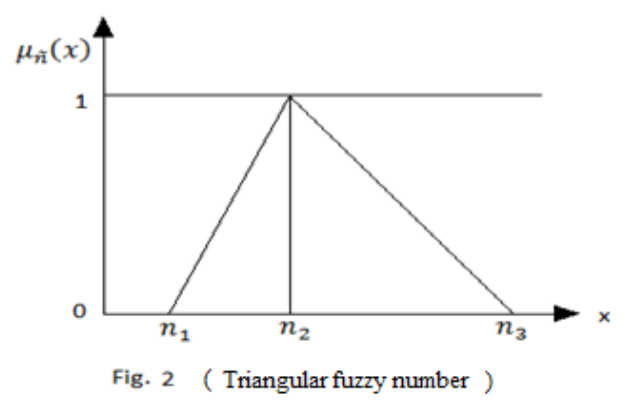

If $n_{1}=n_{2}$ or $n_{2}=n_{3}$ then by convention $\left(n_{1}, n_{2}, n_{3}\right)$ denotes the triangular fuzzy number in figure -3 and any real number $n$ can be represented as the triangular fuzzy number $(n, n, n)$.

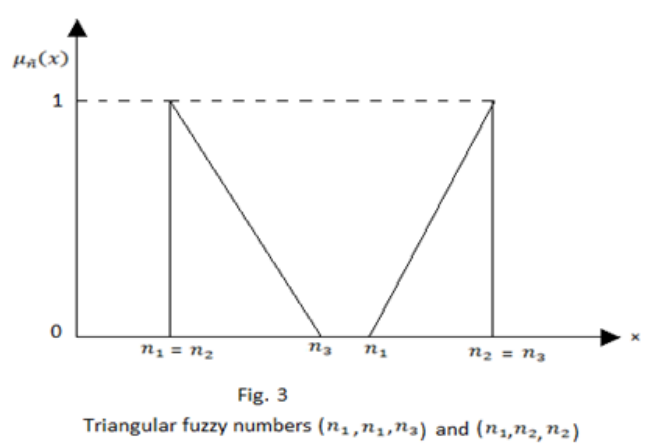

Some operations of fuzzy numbers are defined as follows:

Let $\widetilde{A}=\left(a_{1}, b_{1}, c_{1}\right)$ and $\widetilde{B}=\left(a_{2}, b_{2}, c_{2}\right)$ be two positive triangular fuzzy numbers and $r$ be any real number then

(i) $\widetilde{A}(+) \widetilde{B}=\left(a_{1}+a_{2}, b_{1}+b_{2}, c_{1}+c_{2}\right)$

(ii) $\widetilde{A}(-) \widetilde{B}=\left(a_{1}-a_{2}, b_{1}-b_{2}, c_{1}-c_{2}\right)$

(iii) $\widetilde{A}\left(^{\circ}\right) \widetilde{B}=\left(a_{1} \cdot a_{2}, b_{1} \cdot b_{2}, c_{1} \cdot c_{2}\right)$

(iv) $\mathrm{r}\left(^{\circ}\right) \widetilde{A}=\left(r . a_{1}, r . b_{1}, r . c_{1}\right)$

(v) $\widetilde{A} \emptyset \widetilde{B}=\left(a_{1} / c_{2}, b_{1} / b_{2}, c_{1} / a_{2}\right)$

Definition 7 [39] A matrix is said to be fuzzy matrix if at least one entry is fuzzy number (J.J.Buckley, 1985).

Definition 8 [7] A linguistic variable is the variable which is expressed in terms of some words or sentences. The concept of linguistic variable is very useful in dealing with situation which aretoo complex or too ill defined to be reasonably described in conventional quantitative expression. For example, 'weight' is a linguistic variable whose values are 'very low', 'low', 'medium', 'high' 'very high' etc.

Definition 9 [22] Let $\widetilde{m}=\left(m_{1}, m_{2}, m_{3}\right)$ and $\tilde{n}=$ $\left(n_{1}, n_{2}, n_{3}\right)$ be two triangular fuzzy numbers. If $\tilde{m}=\tilde{n}$ then $m_{i}=n_{i} \forall i=1,2,3$.

Definition 10 [21] Let $\widetilde{m}=\left(m_{1}, m_{2}, m_{3}\right)$ and $\tilde{n}=$ $\left(n_{1}, n_{2}, n_{3}\right)$ be two triangular fuzzy numbers. Then the distance (vertex distance) between two triangular fuzzy numbers is defined as

$$
\frac{d(\tilde{m}, \tilde{n})=}{\sqrt{\frac{1}{3}\left\{\left(m_{1}-n_{1}\right)^{2}+\left(m_{2}-n_{2}\right)^{2}+\left(m_{3}-n_{3}\right)^{2}\right\}}}
$$

Given the fuzzy sets $\widetilde{A}, \widetilde{B}$ and $\widetilde{C}$, the fuzzy set $\widetilde{B}$ is closer to $\widetilde{A}$ than $\widetilde{C}$ if and only if, $d(\widetilde{A}, \widetilde{B})<$ $d(\widetilde{A}, \widetilde{C})$.

Definition: 11.[22] A new distance measure between fuzzy numbers is defined by S. Saghafian et al., using the fuzzy comparison function, which is used in fuzzy TOPSIS method. Suppose fuzzycmp $(\tilde{m}, \tilde{n})$ represents the fuzzy comparison function of two given discrete fuzzy numbers ( $\tilde{m}$ and $\tilde{n})$. Then it has been define that the fuzzycmp $(\widetilde{m}, \tilde{n})$ be the truth of that the fuzzy number $\widetilde{m}$ begreater than or equal to the fuzzy number $\tilde{n}$ Using fuzzylogic, it can be logically defined as:

$\operatorname{fuzzycmp}(\tilde{m}, \tilde{n})=$

$\max \left[\min \left\{\mu_{\tilde{m}}\left(m_{i}\right), \mu_{\tilde{n}}\left(n_{j}\right)\right\}\right], \forall i, j: m_{i} \geq n_{j} \quad$ where $m_{i}$ and $n_{j}$ are the universe element of discrete fuzzy numbers $\widetilde{m}$ and $\widetilde{n}$ respectively then the distance of two fuzzy numbers $\widetilde{m}$ and $\tilde{n}$ is defined as $d(\widetilde{m}, \tilde{n})=$ $|\operatorname{fuzzycmp}(\widetilde{m}, \tilde{n})-\operatorname{fuzzycmp}(\tilde{n}, \widetilde{m})|$. The above distance measure describe the idea that more closer two fuzzy numbers be, more nearer are the truths of one being greater than or equal to the other.

Definition 12 [40] The metric distance ranking technique was proposed by Chen and Cheng (2005) to order fuzzy numbers. Let two fuzzy numbers $A$ and $B$ be defined as follows:

$$
\begin{aligned}
& f_{A}(x)=\left\{\begin{array}{l}
f_{A}^{L}(x), x<m_{A} \\
f_{A}^{R}(x), x<m_{A}
\end{array}\right. \\
& f_{B}(x)=\left\{\begin{array}{l}
f_{B}^{L}(x), x<m_{B} \\
f_{B}^{R}(x), x<m_{B}
\end{array}\right.
\end{aligned}
$$

$m_{A}$ and $m_{B}$ represent the mean of fuzzy numbers $A$ and $B$ respectively. The metric distance between $A$ and $B$ can be calculated as follows

$\mathrm{D}(A, B)=$

$\left[\int_{0}^{1}\left(g_{A}^{L}(y)-g_{B}^{L}(y)\right)^{2} d y+\int_{0}^{1}\left(g_{A}^{R}(y)-g_{B}^{R}(y)\right)^{2} d y\right]$

$g_{A}^{L}, g_{A}^{R}, g_{B}^{L}$ and $g_{B}^{R}$ are the inverse functions of $f_{A}^{L}, f_{A}^{R}, f_{B}^{L}$ and $f_{B}^{R}$ respectively. A triangular fuzzy number can be represented as a symmetry fuzzy number having two parameters namely, standard deviation $(\sigma)$ and mean $(\mu)$. The membership function of the symmetry fuzzy number $A$ in terms of its standard deviation and mean is given as

$$
f_{A}(x)=\left\{\begin{array}{l}
\frac{x-(\mu-\sigma)}{\sigma}, \text { if }(\mu-\sigma) \leq x \leq \mu \\
\frac{(\mu+\sigma)-x}{\sigma}, \text { if } \mu \leq x \leq \mu+\sigma
\end{array}\right.
$$

For a triangular fuzzy number $\left(a_{1}, a_{2}, a_{3}\right)$, the mean and standard deviation can be determined using the following:

$\sigma=\frac{a_{3}-a_{1}}{2}$ 
$\mu=\frac{a_{1}+2 a_{2}+a_{3}}{4}$

The inverse of $f_{A}^{L}$, and $f_{A}^{R}$ are given as

$g_{A}^{L}(y)=(\mu-\sigma)+\sigma y$

$g_{A}^{R}(y)=(\mu+\sigma)-\sigma y$

Definition 13 [41] Let L ( and R ) be decreasing, shape function from $\mathbf{R}_{+}$to $[0,1]$ with $\mathrm{L}(0)=1 ; \mathrm{L}(x)<1$ for all $x>0 ; \mathrm{L}(x)>0$ for all $x<1 ; \mathrm{L}(1)=0$ or $\mathrm{L}(x)>0$ for all and $\mathrm{L}(+\infty=0)$. Then a fuzzy number $X$ is called of LR-type if for $m, \alpha>0, \beta>0$ in $\mathbf{R}$,

$$
X(x)= \begin{cases}L\left(\frac{m-x}{\alpha}\right) & \text { for } x \leq m \\ R\left(\frac{m-x}{\beta}\right) & \text { for } x \geq m\end{cases}
$$

Where $m$ is called the mean value of $X$ and $\alpha$ and $\beta$ is called the left and right spread, respectively. Symbolically $X$ is denoted by $(m, \alpha, \beta)_{\text {LR }}$.

Now let $F_{L R}(\mathbf{R})$ denote the set of all $L R$-type fuzzy numbers. In order to consider a class of Fuzzy cNumbers (FCNs) [2] on $F_{L R}$ (R) Yang and Cheng defined a new type of distance $d_{L R}$ for any $X$ and $Y$ in $F_{L R}(\mathbf{R})$ with $X=\left(m_{x}, \alpha_{x}, \beta_{x}\right)_{L R}$ and $Y=\left(m_{Y}, \alpha_{Y}, \beta_{Y}\right)_{L R}$ as follows:

$$
\begin{gathered}
d_{L R}^{2}(X, Y)=\left(m_{x}-m_{y}\right)^{2}+\left(\left(m_{x}-l \alpha_{x}\right)-\left(m_{y}-l \alpha_{y}\right)\right)^{2}+ \\
\left(\left(m_{x}+r \beta_{x}\right)-\left(m_{y}+r \beta_{y}\right)\right)^{2} \\
\text { Where } \quad l=\int_{0}^{1} L^{-1}(\omega) d \omega \text { and } r=\int_{0}^{1} R^{-1}(\omega) d \omega .
\end{gathered}
$$

In $L R$-type fuzzy numbers, the triangular and normal fuzzy numbers are most commonly used. For a $L R$-type fuzzy number $X=(m, \alpha, \beta)_{L R}$, if $L$ and $R$ are of the form

$$
T(x)=\left\{\begin{array}{cr}
1-x & \text { for } x \leq m(\alpha>0) \\
0 & \text { otherwise }
\end{array}\right.
$$

then $X$ is called a triangular fuzzy number, denoted by $X=(m, \alpha, \beta)_{L R}$, i.e.

$$
X(x)= \begin{cases}1-\frac{m-x}{\alpha} & \text { for } x \leq m(\alpha>0) \\ 1-\frac{m-x}{\beta} & \text { for } x \geq m(\beta>0)\end{cases}
$$

Consider two triangular fuzzy numbers $X=\left(m_{x}, \alpha_{x}\right.$ $\left.\beta_{x}\right)_{\mathrm{T}}$ and $Y=\left(m_{Y}, \alpha_{Y}, \beta_{Y}\right)_{T}$ of the space $F_{L R}(\mathbf{R})$ which represents the collection of all triangular fuzzy numbers. According to the distance $d_{T}(X, Y)$ on $F_{L R}$ (R) as follows:

$$
\begin{gathered}
d_{L R}^{2}(X, Y)=\left(m_{x}-m_{y}\right)^{2}+\left(\left(m_{x}-\frac{1}{2} \alpha_{x}\right)-\left(m_{y}-\frac{1}{2} \alpha_{y}\right)\right)^{2}+ \\
\left(\left(m_{x}+\frac{1}{2} \beta_{x}\right)-\left(m_{y}+\frac{1}{2} \beta_{y}\right)\right)^{2}
\end{gathered}
$$

Where, $l=r=\frac{1}{2}$.

Definition 14 [2] Tran and Duckstein (2002) introduced an approach for ranking fuzzy numbers based on a distance measure. Tran and Duckstein gave the equations to compute distances for triangular fuzzy numbers with two different weighting functions: $w(\alpha$ )$=1$ and $w(\alpha)=\alpha$. Since triangular fuzzy numbers (TFNs) are the most widely-used fuzzy numbers, this study assumes that a fuzzy number-valued fuzzy set function $\mu(\mathrm{z})$ on fuzzy measure space $\left(X, F^{*}(X), \mu\right)$ is a TFN for simplicity. Given two TFNs that $\mathrm{A}=\left(a_{1}, a_{2}, a_{3}\right)$ and $\mathrm{B}=\left(b_{1}, b_{2}, b_{3}\right)$. When $w(\alpha)=1$, the distance between $A$ and $B$ is as follows:

$\mathrm{D}_{\mathrm{TFN}}=$

$$
\begin{aligned}
& \left(a_{2}-b_{2}\right)^{2}+\frac{1}{2}\left(a_{2}-b_{2}\right)\left[\left(a_{3}+a_{1}\right)-\left(b_{3}+b_{1}\right)\right]+ \\
& \frac{1}{9}\left[\left(a_{3}-a_{2}\right)^{2}+\left(a_{2}-a_{1}\right)^{2}+\left(b_{3}-b_{2}\right)^{2}+\left(b_{2}-b_{1}\right)^{2}\right] \\
& -\frac{1}{9}\left[\left(a_{2}-a_{1}\right)\left(a_{3}-a_{2}\right)+\left(b_{2}-b_{1}\right)\left(b_{3}-b_{2}\right)\right]+ \\
& \frac{1}{6}\left(2 a_{2}-a_{1}-a_{3}\right)\left(2 b_{2}-b_{1}-b_{3}\right) .
\end{aligned}
$$

In this study we apply Tran and Duckstein's distance measure for fuzzy triangular numbers to estimated distance of attribute importance from positive ideal solution and negative ideal solution in TOPSIS.

Definition 15 [42]. Given two fuzzy subsets of $A$ and $B$ with a reference set, $X=\left\{\begin{array}{ll}x_{1}, & x_{2}, \ldots, x_{n}\end{array}\right\}$ and memberships function $\mu_{A}$ and $\mu_{B}$. Then the Hamming distance is defined as

$$
d(A, B)=\sum_{j=1}^{n}\left|\mu_{A}\left(x_{j}\right)-\mu_{B}\left(x_{j}\right)\right|
$$

The Hamming distance for two interval valued fuzzy numbers $\mathrm{A}$ and $\mathrm{B}$, whose membership function are as follows:

$$
\begin{aligned}
& \mu_{A}\left(x_{j}\right)=\left[a_{x_{j}}^{L}, a_{x_{j}}^{U}\right], \\
& \mu_{B}\left(x_{j}\right)=\left[b_{x_{j}}^{L}, b_{x_{j}}^{U}\right]: j=1,2,3, \ldots \ldots, n \\
& \text { is defined as } d_{H D}=\left(\sum_{j=1}^{n}\left(\left|a_{x_{j}}^{L}-b_{x_{j}}^{L}\right|\right)+\left(\left|a_{x_{j}}^{U}-b_{x_{j}}^{U}\right|\right)\right) .
\end{aligned}
$$

Major headings should be typeset in boldface with the words uppercase.

\section{AN ANALYTICAL STUDY OF TOPSIS METHOD USING SIX DIFFERENT DISTANCE MEASURES:}

A decision making problem is a process to finding the best option among the set of feasible alternative. A multicriteria decision making (MCDM) problem can be expressed in the matrix format as follows:[1] 


\section{Available online at www.ijrat.org}

$$
\begin{gathered}
C_{1} \\
A_{1} \\
A_{2} \\
\vdots \\
A_{n}
\end{gathered} \quad\left[\begin{array}{ccccc}
\tilde{x}_{11} & \tilde{x}_{12} & \ldots \ldots \ldots & \tilde{x}_{1 n} \\
\tilde{x}_{21} & \tilde{x}_{22} & \ldots \ldots . . & \tilde{x}_{2 n} \\
\ldots \ldots & \ldots \ldots & \ldots \ldots . . & \ldots \ldots \\
\tilde{x}_{m 1} & \tilde{x}_{m 2} & \ldots \ldots . . & \tilde{x}_{m n}
\end{array}\right]
$$

where $A_{1}, A_{2}, \ldots, A_{n}$ are possible alternatives, $C_{1}, C_{2}, \ldots, C_{m}$ are criteria of the alternatives and $\tilde{x}_{i j}$ is the rating of alternative $A_{i}$ with respect to criteria $C_{j}$, also let $\widetilde{w}_{j}$ describes the weight of the criteria $C_{j}$.

Linguistic variables which are used to defined the weights of various criteria and the ratings of many qualitative criteria are expressed in terms of positive triangular fuzzy numbers (shown in table I and II in numerical example). When the decision makers use the linguistic variables to evaluate the weights of different criteria and ratings of alternative with respect to various criteria the following calculations are adopted treating the decision group has $\mathrm{K}$ persons.

$$
\begin{aligned}
\tilde{x}_{i j} & =\frac{1}{K}\left[\tilde{x}_{i j}^{1}(+) \tilde{x}_{i j}^{2}(+) \ldots \ldots(+) \tilde{x}_{i j}^{K}\right] \\
\widetilde{w}_{j} & =\frac{1}{K}\left[\widetilde{w}_{j}^{1}(+) \widetilde{w}_{j}^{2}(+) \ldots \ldots(+) \widetilde{w}_{j}^{K}\right]
\end{aligned}
$$

Where $\tilde{x}_{i j}^{K}$ and $\widetilde{w}_{j}^{K}$ are the ratings and important weight of the $\mathrm{K}^{\text {th }}$ decision maker and (+) indicates the fuzzy arithmetic summation function. The new weight vector can be written as

$$
\tilde{w}_{j}=\left[\begin{array}{llll}
\tilde{w}_{1} & \tilde{w}_{2} & \ldots \ldots & \tilde{w}_{n}
\end{array}\right]
$$

Where $\tilde{x}_{i j}$ and $\tilde{w}_{j}$ are linguistic variable which are expressed in triangular fuzzy numbers: $\tilde{x}_{i j}=\left(a_{i j}, b_{i j}, c_{i j}\right)$ and $\tilde{w}_{j}=\left(\begin{array}{lllll}w_{j 1} & , & w_{j 2} & , & w_{j 3}\end{array}\right)$

To avoid the complicated normalization formula used in classical TOPSIS [10], linear scale transformation can be used to transform the various criteria scale into a comparable scale. So it is possible to obtain the normalized fuzzy decision matrix denoted by $\widetilde{R}$.

$$
\widetilde{R}=\left[\tilde{r}_{i j}\right]_{m \times n}
$$

Where $\mathrm{B}$ and $\mathrm{C}$ are the set of benefit criteria and cost criteria and

$$
\begin{aligned}
\tilde{r}_{i j} & =\left(\frac{a_{i j}}{c_{j}^{*}}, \frac{b_{i j}}{c_{j}^{*}}, \frac{c_{i j}}{c_{j}^{*}}\right), \quad j \in B \\
\tilde{r}_{i j} & =\left(\frac{a_{j}^{-}}{c_{i j}}, \frac{a_{j}^{-}}{b_{i j}}, \frac{a_{j}^{-}}{a_{i j}}\right), \quad j \in C \\
c_{j}^{*} & =\max _{i} c_{i j} \quad, \text { if } j \in B \\
a_{j}^{-} & =\min _{i} a_{i j} \quad, \text { if } j \in C
\end{aligned}
$$

The normalized method mention above is to preserve the property that the ranges of normalized triangular fuzzy numbers belong to $[0,1]$. In this study the above normalization method is avoided because the fuzzy numbers taken are in the interval

[0,1]. Therefore we have $\tilde{r}_{i j}=\tilde{x}_{i j}$ and $\tilde{R}=\widetilde{D}$.

Now one can construct the weighted normalized fuzzy decision matrix according as follows:

$$
\widetilde{V}=\left[\tilde{v}_{i j}\right]_{m \times n} i=1,2, \ldots \ldots m, j=1,2, \ldots \ldots n
$$

Where $\tilde{v}_{i j}=\tilde{r}_{i j}(\cdot) \widetilde{w}_{j}$ and are normalized positive triangular fuzzy numbers. Then Fuzzy Positive Ideal Solution (FPIS, $A^{*}$ ) and Fuzzy Negative Ideal Solution (FNIS, $A^{-}$) are defined as follows:

$$
\begin{gathered}
A^{*}=\left(\tilde{v}_{1}^{*}, \tilde{v}_{2}^{*}, \ldots \ldots, \tilde{v}_{n}^{*}\right) \\
A^{-}=\left(\tilde{v}_{1}^{-}, \tilde{v}_{2}^{-}, \ldots \ldots, \tilde{v}_{n}^{-}\right)
\end{gathered}
$$

Where $\tilde{v}_{j}^{*}=(1.0,1.0,1.0)$ and $\tilde{v}_{j}^{-}=(0.0,0.0,0.0)$.

The distance of each alternative $A_{i}=(i=1,2, \ldots \ldots m)$ from $A^{*}$ and $A^{-}$can be calculated as follows:

$$
\begin{aligned}
& d_{i}{ }^{*}=\sum_{j=1}^{n} d\left(\tilde{v}_{i j}, \tilde{v}_{j}^{*}\right), \forall i=1,2, \ldots \ldots m \\
& d_{i}{ }^{-}=\sum_{j=1}^{n} d\left(\tilde{v}_{i j}, \tilde{v}_{j}^{-}\right), \forall i=1,2, \ldots \ldots n
\end{aligned}
$$

Where, $d(\cdot, \cdot)$ is the distance measure between two fuzzy numbers. Then the closeness coefficient of each alternative is calculated as:

$$
C C_{i}=\frac{d_{i}^{-}}{d_{i}^{*}+d_{i}^{-}}, \forall i=1,2, \ldots \ldots m .
$$

Based on the above equation an alternative $A_{i}$ would be closer to FPIS and further from FNIS as $C C_{i}$ approaches 1. On the basis of the closeness coefficient $C C_{i}$ the best alternative is chosen. Here in our discussion we shall use the distance measures defined in definition 10 to 15 throughout in an example and one conclusion is to be made to obtain a preferable distance measure for TOPSIS which gives closer closeness coefficient value to one. To demonstrate and make use of the approach the following numerical example is carried.

\section{NUMERICAL EXAMPLE: [22]}

Assume that university " $\mathrm{X}$ " desires to hire a professor for teaching fuzzy theory course. A committee of three expert decision makers, D1, D2 and D3 has been formed to conduct the interview with three eligible candidates, namely $\mathrm{A} 1, \mathrm{~A} 2$ and $\mathrm{A} 3$, and to select the most suitable candidate. Five benefit criteria are considered: (1) Publications and researches (C1), (2) Teaching skills (C2), (3) Practical experiences in industries and corporations (C3), (4) Past experiences in teaching (C4), (5) Teaching discipline (C5).

\section{Computational procedure is discussed in detail} below:

Step 1: Decision makers choose the linguistic weighting variable (Table 1)for the importance weight of criteria and the linguistic ratings variable (Table 2) to evaluate the ratings of alternatives with respect to each criterion.

Table 1: Linguistic Variable for the Importance Weight of each Criterion

\begin{tabular}{|l|l|}
\hline Very Low $(\mathrm{VL})$ & $(0.0,0.0,0.1)$ \\
\hline Low $(\mathrm{L})$ & $(0.0,0.1,0.25)$ \\
\hline Medium Low (ML) & $(0.15,0.3,0.45)$ \\
\hline Medium (M) & $(0.35,0.5,0.65)$ \\
\hline
\end{tabular}


International Journal of Research in Advent Technology, Vol.7, No.5, May 2019

E-ISSN: 2321-9637

Available online at www.ijrat.org

\begin{tabular}{|l|l|}
\hline Medium High $(\mathrm{MH})$ & $(0.55,0.7,0.85)$ \\
\hline High $(\mathrm{H})$ & $(0.8,0.9,1.0)$ \\
\hline Very High $(\mathrm{VH})$ & $(0.9,1.0,1.0)$ \\
\hline
\end{tabular}

Table 2: Linguistic Variables For The Ratings

\begin{tabular}{|l|l|}
\hline Very Poor (VP) & $(0.0,0.0,0.1)$ \\
\hline Poor (P) & $(0.0,0.1,0.2)$ \\
\hline Medium Poor (MP) & $(0.2,0.3,0.5)$ \\
\hline Fair (F) & $(0.35,0.5,0.65)$ \\
\hline Medium Good (MG) & $(0.5,0.7,0.9)$ \\
\hline Good (G) & $(0.65,0.8,0.95)$ \\
\hline Very Good $(\mathrm{VG})$ & $(0.9,1.0,1.0)$ \\
\hline
\end{tabular}

Step 2: To assess the important of the criteria (Table 3) linguistic weighting variable are used (Table 1)

Table 3: The Importance Weight Of Each Criterion Given By Decision Makers For The Numerical:

\begin{tabular}{|l|l|l|l|}
\hline $\begin{array}{c}\text { Decision } \\
\text { makers }\end{array}$ & D1 & D2 & D3 \\
\hline C1 & H & VH & MH \\
\hline C2 & VH & VH & VH \\
\hline C3 & VH & H & H \\
\hline C4 & VH & VH & VH \\
\hline C5 & M & MH & MH \\
\hline
\end{tabular}

Step 3: The weights of criteria are aggregated using equation (1) to get the aggregated fuzzy weight $\widetilde{w}_{j}$ of the criterion $C_{j}$ and decision makers gave their opinion (Table 4 ) to get the aggregated fuzzy ratings $\tilde{x}_{i j}$ of alternative $A_{i}$ under criterion $C_{j}$.

$$
\widetilde{w}_{\mathrm{j}}=\frac{1}{K}\left[\widetilde{w}_{\mathrm{j}}^{1}(+) \widetilde{w}_{\mathrm{j}}^{2}(+) \ldots \ldots(+) \widetilde{w}_{\mathrm{j}}^{K}\right]
$$

$$
\begin{aligned}
& \widetilde{w}_{1}= \\
& \frac{1}{3}[(0.8,0.9,1.0)(+)(0.9,1.0,1.0)(+)(0.55,0.7,0.85)] \\
& =(0.75, \quad 0.87,0.95) \\
& \widetilde{w}_{2} \\
& =\frac{1}{3}[(0.9,1.0,1.0)(+)(0.9,1.0,1.0)(+)(0.9,1.0,1.0)] \\
& =(0.9,1.0,1.0) \\
& \widetilde{w}_{3} \\
& =\frac{1}{3}[(0.9,1.0,1.0)(+)(0.8,0.9,1.0)(+)(0.8,0.9,1.0)] \\
& =(0.83,0.93,1.0) \\
& \widetilde{w}_{4} \\
& =\frac{1}{3}[(0.9,1.0,1.0)(+)(0.9,1.0,1.0)(+)(0.9,1.0,1.0)] \\
& =(0.9,1.0,1.0)
\end{aligned}
$$

$$
\begin{aligned}
& \widetilde{w}_{5}=\frac{1}{3}\left[\begin{array}{c}
(0.35,0.5,0.65)(+)(0.55,0.7,0.85)(+) \\
(0.55,0.7,0.85)
\end{array}\right] \\
= & (0.48, \quad 0.63,0.78)
\end{aligned}
$$

Table - 4 The Final Aggregate Result Obtained From Ratings Given By Decision Makers:

\begin{tabular}{|l|l|l|}
\hline \multirow{2}{*}{ Criterion } & Alternative & $\begin{array}{l}\text { Linguistic } \\
\text { variable }\end{array}$ \\
\hline \multirow{4}{*}{ C1 } & A1 & MG \\
\cline { 2 - 3 } & A2 & G \\
\cline { 2 - 3 } & A3 & VG \\
\hline \multirow{4}{*}{ C2 } & A1 & G \\
\cline { 2 - 3 } & A2 & VG \\
\cline { 2 - 3 } & A3 & MG \\
\hline \multirow{4}{*}{ 3 } & A1 & F \\
\cline { 2 - 3 } & A2 & VG \\
\cline { 2 - 3 } & A3 & G \\
\hline \multirow{4}{*}{ C4 } & A1 & VG \\
\cline { 2 - 3 } & A2 & VG \\
\cline { 2 - 3 } & A3 & G \\
\hline \multirow{4}{*}{ C5 } & A1 & F \\
\cline { 2 - 3 } & A2 & VG \\
\cline { 2 - 3 } & A3 & G \\
\hline
\end{tabular}

Step 4: The fuzzy decision matrix $\widetilde{D}$ is constructed as follows using table 4 . Since all the weights and ratings are in the interval $[0,1]$, so the matrix $\widetilde{D}$ becomes the normalised fuzzy decision matrix.

$\widetilde{D}=$

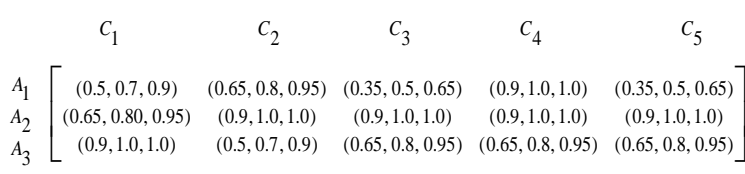

Step.5: The weighted normalized fuzzy decision matrix is now constructed by using equation (2).

$\widetilde{D}=$

$$
\begin{array}{cccccc}
C_{1} & C_{2} & C_{3} & C_{4} & C_{5}
\end{array}
$$

$A_{1} \quad\left[\begin{array}{lllll}(0.375,0.609,0.855) & (0.585,0.8,0.95) & (0.291,0.465,0.65) & (0.81 .1 .0,1.0) & (0.168,0.315,0.507)\end{array}\right]$

$A_{2} \quad\left(\begin{array}{llllll} & (0.488,0.696,0.903) & (0.81,1.0,1.0)) & (0.0 .747,0.93,1.0) & (0.81,1.0,1.0) & (0.432,0.63,0.78)\end{array}\right.$

$A_{2}$
$A_{3}$$\left[\begin{array}{ccccc}(0.488,0.675,0.87,0.95) & (0.45,0.7,0.9) & (0.540,0.744,0.95) & (0.585,0.8,0.95) & (0.312,0.504,0.741)\end{array}\right]$

Step 6: FPIS and FNIS are defined as:

$$
\begin{aligned}
& \tilde{A}^{*}=\left[\begin{array}{lllll}
(1,1,1) & (1,1,1) & (1,1,1) & (1,1,1) & (1,1,1)
\end{array}\right] \\
& \equiv\left(\tilde{v}_{1}^{*}, \tilde{v}_{2}^{*}, \tilde{v}_{3}^{*}, \tilde{v}_{4}^{*}, \tilde{v}_{5}^{*}\right) \\
& \tilde{A}^{-}=\left[\begin{array}{lllll}
(0,0,0) & (0,0,0) & (0,0,0) & (0,0,0) & (0,0,0)
\end{array}\right] \\
& \equiv\left(\tilde{v}_{1}^{-}, \tilde{v}_{2}^{-}, \tilde{v}_{3}^{-}, \tilde{v}_{4}^{-}, \tilde{v}_{5}^{-}\right)
\end{aligned}
$$

Step 7: In this step, different distance measures are used to determine the closeness coefficients of each alternative in order to obtain a order ranking of the alternatives..

The distances of each candidate from FPIS and FNIS are calculated by using the distance measure defined in 


\section{Available online at www.ijrat.org}

definition 11 and the closeness coefficient are obtained as follows.

$C C_{1}=0.56, C C_{2}=0.82, C C_{3}=0.69$

Therefore the order ranking is $A_{2}>A_{3}>A_{1}$.

Now, we calculate the above closeness coefficient by using a distance measure between two fuzzy numbers which is defined in definition 10 .

The closeness coefficient are obtained as follows: $C C_{1}=0.63, C C_{2}=0.77, C C_{3}=0.69$.

The order ranking is obtained as $A_{2}>A_{3}>A_{1}$.

Again we apply the metric distance measure for order ranking the alternatives defined in definition 12.as follows:

$\mathrm{D}(A, B)=$

$\left[\int_{0}^{1}\left(g_{A}^{L}(y)-g_{B}^{L}(y)\right)^{2} d y+\int_{0}^{1}\left(g_{A}^{R}(y)-g_{B}^{R}(y)\right)^{2} d y\right]$

$g_{A}^{L}(y)=(\mu-\sigma)+\sigma y$ and

$g_{A}^{R}(y)=(\mu+\sigma)-\sigma y$

$\sigma=\frac{a_{3}-a_{1}}{2} \quad \mu=\frac{a_{1}+2 a_{2}+a_{3}}{4}$

The closeness coefficient is calculated:

$C C_{1}=0.6925, C C_{2}=0.9236, C C_{3}=0.8338$.

The order ranking is obtained as $A_{2}>A_{3}>A_{1}$.

Now we apply the distance measure defined by Tran and Duckstein in definition 14. Let $A=\left(a_{1}, a_{2}, a_{3}\right)$ and $B=\left(b_{1}, b_{2}, b_{3}\right)$ be two triangular fuzzy numbers then

$\mathrm{D}_{\mathrm{TFN}}=$

$$
\begin{aligned}
& \left(a_{2}-b_{2}\right)^{2}+\frac{1}{2}\left(a_{2}-b_{2}\right)\left[\left(a_{3}+a_{1}\right)-\left(b_{3}+b_{1}\right)\right] \\
& +\frac{1}{9}\left[\left(a_{3}-a_{2}\right)^{2}+\left(a_{2}-a_{1}\right)^{2}+\left(b_{3}-b_{2}\right)^{2}+\left(b_{2}-b_{1}\right)^{2}\right] \\
& -\frac{1}{9}\left[\left(a_{2}-a_{1}\right)\left(a_{3}-a_{2}\right)+\left(b_{2}-b_{1}\right)\left(b_{3}-b_{2}\right)\right] \\
& +\frac{1}{6}\left(2 a_{2}-a_{1}-a_{3}\right)\left(2 b_{2}-b_{1}-b_{3}\right) .
\end{aligned}
$$

Using this distance measure we obtained the closeness coefficients as: $C C_{1}=0.7057, C C_{2}=0.9354, C C_{3}=$ 0.8487 .

Ranking of the alternatives are $A_{2}>A_{3}>A_{1}$.

Next we apply the distance measure defined in definition 13 as

$$
\begin{aligned}
& d_{L R}^{2}(X, Y)= \\
& \left(m_{x}-m_{y}\right)^{2}+\left(\left(m_{x}-\frac{1}{2} \alpha_{x}\right)-\left(m_{y}-\frac{1}{2} \alpha_{y}\right)\right)^{2} \\
& +\left(\left(m_{x}+\frac{1}{2} \beta_{x}\right)-\left(m_{y}+\frac{1}{2} \beta_{y}\right)\right)^{2}
\end{aligned}
$$

The closeness coefficient is calculated as follows: $C C_{1}=0.7432, C C_{2}=0.9651, C C_{3}=0.9038$.

Thus, it has been seen that the order ranking of alternatives for all the distance measures is $\mathrm{A} 2>\mathrm{A} 3>$ A1.

Here, we now apply the Hamming distance to determine the ranking order of the alternatives as

\begin{tabular}{|c|c|c|c|c|c|c|}
\hline & $\begin{array}{l}\alpha= \\
0.1\end{array}$ & $\begin{array}{l}\alpha= \\
0.3\end{array}$ & $\begin{array}{l}\alpha= \\
0.5\end{array}$ & $\begin{array}{l}\alpha= \\
0.7\end{array}$ & $\begin{array}{l}\alpha= \\
0.9\end{array}$ & $\begin{array}{l}\text { Averag } \\
\text { e } \mathrm{CC}_{3}\end{array}$ \\
\hline $\mathrm{d}$ & 2.928 & 2.892 & 2.855 & 2.818 & 2.782 & \multirow{3}{*}{$\begin{array}{l}0.7144 \\
5\end{array}$} \\
\hline+ & 7 & 1 & 5 & 9 & 3 & \\
\hline $\mathrm{d}^{-}$ & $\begin{array}{l}7.071 \\
\end{array}$ & $\begin{array}{l}7.107 \\
9\end{array}$ & $\begin{array}{l}7.144 \\
5\end{array}$ & 7.181 & $\begin{array}{l}7.217 \\
7\end{array}$ & \\
\hline
\end{tabular}
defined in definition 15.

For the first alternative: Table-5 (i)

\begin{tabular}{|l|l|l|l|l|l|l|}
\hline & $\begin{array}{l}\alpha= \\
0.1\end{array}$ & $\begin{array}{l}\alpha=3 \\
0.3\end{array}$ & $\begin{array}{l}\alpha= \\
0.5\end{array}$ & $\begin{array}{l}\alpha= \\
0.7\end{array}$ & $\begin{array}{l}\alpha= \\
0.9\end{array}$ & $\begin{array}{l}\text { Averag } \\
\mathrm{e} \mathrm{CC}_{1}\end{array}$ \\
\hline $\begin{array}{c}\mathrm{d} \\
+\end{array}$ & $\begin{array}{l}3.790 \\
3\end{array}$ & $\begin{array}{l}3.752 \\
9\end{array}$ & $\begin{array}{l}3.715 \\
5\end{array}$ & $\begin{array}{l}3.678 \\
1\end{array}$ & $\begin{array}{l}3.640 \\
7\end{array}$ & $\begin{array}{l}0.6284 \\
5\end{array}$ \\
\cline { 1 - 5 } $\mathrm{d}^{-}$ & $\begin{array}{l}6.209 \\
7\end{array}$ & $\begin{array}{l}6.247 \\
1\end{array}$ & $\begin{array}{l}6.284 \\
5\end{array}$ & $\begin{array}{l}6.321 \\
9\end{array}$ & $\begin{array}{l}6.959 \\
3\end{array}$ & \\
\hline
\end{tabular}

For the second alternative: (ii)

\begin{tabular}{|l|l|l|l|l|l|l|}
\hline & $\alpha=$ & $\alpha=$ & $\alpha=$ & $\alpha=$ & $\alpha=$ & Averag \\
& 0.1 & 0.3 & 0.5 & 0.7 & 0.9 & ${\text { e } C_{2}}^{2}$ \\
\hline $\mathrm{d}$ & 1.975 & 1.867 & 1.75 & 1.650 & 1.542 & 0.8241 \\
+ & 8 & 4 & 9 & 6 & 2 & \\
\cline { 1 - 5 } $\mathrm{d}^{-}$ & 8.024 & 8.132 & 8.24 & 8.349 & 8.457 & \\
& 2 & 6 & 1 & 4 & 8 & \\
\hline
\end{tabular}

For the third alternative: (iii)

From the table 5 (i), (ii), (iii) the order ranking of the alternative are $\mathrm{A} 2>\mathrm{A} 3>\mathrm{A} 1$.

\section{DISCUSSION AND CONCLUSION:}

Fuzzy TOPSIS is based on the concept that the chosen alternative should have the shortest distance from the positive-ideal solution and the longest distance from the negative-ideal solution. The higher value of closeness coefficient indicates that an alternative is closer to fuzzy PIS and farther from fuzzy NIS simultaneously, i.e., an alternative $A_{i}$ be treated closer to FPIS and further from FNIS as closeness coefficient $\left(C C_{i}\right)$ approaches to one. In this paper, we have compared the different results of closeness coefficient obtained by using different distance measures in fuzzy TOPSIS procedure. The following table- 6 shows the different values of closeness coefficient of different alternatives regarding the respective distance measures.

Table-6

\begin{tabular}{|l|l|l|l|l|}
\hline Rank & $\begin{array}{l}\text { Distance } \\
\text { measure } \\
(\downarrow)\end{array}$ & $\mathrm{CC}_{1}$ & $\mathrm{CC}_{2}$ & $\mathrm{CC}_{3}$ \\
\hline 1 & L-R & 0.7432 & 0.9651 & 0.9038 \\
\hline 2 & $\begin{array}{l}\text { Tran \& } \\
\text { Duckstein }\end{array}$ & 0.7057 & 0.9354 & 0.8487 \\
\hline 3 & Metric & 0.6925 & 0.9236 & 0.8338 \\
\hline
\end{tabular}


Available online at www.ijrat.org

\begin{tabular}{|l|l|l|l|l|}
\hline & Distance & & & \\
\hline 4 & Fuzzycmp & 0.56 & 0.82 & 0.69 \\
\hline 5 & Vertex & 0.63 & 0.77 & 0.69 \\
\hline 6 & $\begin{array}{l}\text { Hamming } \\
\text { Distance }\end{array}$ & 0.62845 & 0.8241 & 0.71445 \\
\hline
\end{tabular}

The order raking of usefulness of various distances measures in TOPSIS have shown graphically in figure 4.

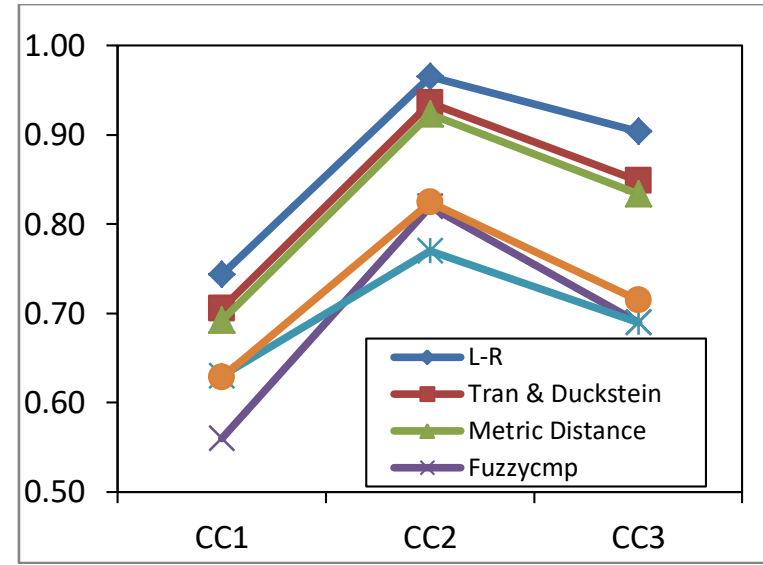

Figure-4 (Graphical representation )

The analyzed results show that use of the L-R distance measure in TOPSIS for order ranking gives the higher value of closeness coefficient followed by while using the Tran and Duckstein distance measure, metric distance measure and the distance measure defined by Saghafian et al. and the well-known Hamming and vertex distance measure. Though different distance measures are used in TOPSIS method, there is no counterintuitive case encountered during the ranking process of the alternatives with respect different criteria. This comparative study reflects the reliability and stability of TOPSIS method and hence can be implemented in various fuzzy decision making process in order to obtain a proper ranking of alternatives.

\section{REFERENCES}

[1] Hwang, C.L., and Yoon, K. (1981), Multiple Attribute Decision Making Method and application, Heidelberg Verlag Berlin: Springer.

[2] Tran, L., and Duckstein, L. (2002), Comparison of fuzzy numbers using a fuzzy distance measure, Fuzzy Sets and Systems, 130 (3), 331-341.

[3] Cheng, C.H. (1998), A new approach for ranking fuzzy numbers by distance method, Fuzzy Sets and Systems, 95(3), 307-317.

[4] Voxman, W. (1998), Some remarks on distances between fuzzy numbers, Fuzzy Sets and Systems, 100, 353-365

[5] Diamond, P. (1988), Fuzzy least squares, Information Sciences, 46, 141-157.

[6] Chakraborty, C., and Chakraborty, D. (2006), A theoretical development on a fuzzy distance measure for fuzzy numbers, Mathematical and Computer Modelling, 43, 254-261.

[7] Zadeh, L.A. (1965), Fuzzy sets, Inform. and Contro., 8, 338-353.

[8] Bellman, R.E., and Zadeh, L.A. (1970), Decision-Making in a Fuzzy Environment, Management science, 17(4), B-141-B-164.

[9] Zimmermann, H.J. (1987), Fuzzy Sets, Decision Making, and Expert Systems, Boston; Kluwer Academic Publishers.

[10]Baas, S.M., and Kwakernaak, H. (1977), Rating and ranking of multiple-aspect alternatives using fuzzy sets, Automatica, 13, 47-58.

[11] Yager, R.R. (1978), Fuzzy decision making including unequal objectives, Fuzzy Sets and Systems, 1, 87-95.

[12] Chen, S.J., and Hwang, C.L., (1992), Fuzzy Multiple Attribute decision-making Methods and Applications, Verlag Berlin Heidelberg: Springer.

[13] Kickert, W.J.M., (1978), Towards an analysis of linguistic modelling, Fuzzy Sets and Systems, 2(4), 293-308.

[14] Dubois, D., and Prade, H. (1980), Fuzzy Sets and Systems Theory and Applications, New York: Academic Press.

[15]Zimmermann, H.J. (1987), Fuzzy Sets, Decision Making, and Expert Systems, Boston: Kluwer Academic Publishers.

[16] Riberio, R.A. (1996), Fuzzy multiple attribute decision making, a review and new reference elicitation techniques, Fuzzy Sets and Systems, 78, 155-181.

[17]Liang, G.S. (1999), Fuzzy MCDM based on ideal and anti-ideal concepts. European Journal of Operational Research, 11, 682-691.

[18] Lai, Y.J., Liu, T.Y, and Hwang, C.L. (1994), TOPSIS for MODM, European Journal of Operational Research, 76, 486-500.

[19] Yoon, K.P., and Hwang, C. (1995), Multiple Attribute Decision Making An introduction, Thousand oaks CA: SAGE publications Inc. 104.

[20] Triantaphyllou, E., and Lin, C.T. (1996), Development and evaluation of five fuzzy multiattribute decision-making methods, International Journal of Approximate Reasoning, 14, 281-310.

[21] Chen, C.T. (2000), Extension of the TOPSIS for Group Decision Making Under Fuzzy Environment, Fuzzy Sets and System, 114, 19.

[22] Saghafian, S., and Hejazi, S.R. (2005), Multicriteria group decision making using a modified TOPSIS procedure, IEEE, 2, 215221.

[23] Abo-Sinna, M.A., and Abou-El-Enien, T.H.M. (2006), An interactive algorithm for large scale multiple objective programming problems 
with fuzzy parameters through TOPSIS approach, Applied Mathematics and Computation, forthcoming, 117(2), 515-527.

[24] Wang, Y.M., and Elhag, T.M.S. (2006), Fuzzy TOPSIS method based on alpha level sets with an application to bridge risk assessment, Expert Systems with Applications, 31, 309-319.

[25] Jahanshahloo, G.R., Hosseinzadeh, L.F., and Izadikhah, M. (2006), An algorithmic method to extend TOPSIS for decision-making problems with interval data, Applied Mathematics and Computation, 175, 13751384.

[26] Ashtiani, B., Haghighirad, F., and Makui, A. (2009), Extension of Fuzzy TOPSIS method based on interval-values fuzzy sets, Applied Soft Computing, 9, 457-461.

[27] Chen, S.M., and Lee, L.W. (2010), Fuzzy multiple attributes group decision-making based on the interval type-2 TOPSIS method, Expert systems with applications, 37, 27902798.

[28] Yang, T., and Hung, C.C. (2007), Multipleattribute decision making methods for plant layout design problem, Robotics and Computer-Integrated Manufacturing, 23, 126137.

[29] Wang, Y.J., and Lee, H.S. (2007), Generalizing TOPSIS for fuzzy multiplecriteria group decision-making, Comput. Math. Appl.,53,1762-1772.

[30]Chen, T.Y., and Tsao, C.Y. (2008), The interval-valued fuzzy TOPSIS method and experimental analysis, Fuzzy Sets and Systems, 159, 1410-1428.

[31][31] Sun, C.C. (2010), A Performance Evaluation Model by Integrating Fuzzy AHP and Fuzzy TOPSİS Methods, Expert Systems with Applications,37, 7745-7754.

[32] Brans, J.P., and Vincke. (1985), A preference ranking organization method, Management Science, 31, 647-656.

[33]El-Hawary, M.E. (1998), Electric Power Applications of Fuzzy Systems, The Institute of Electrical and Electronics Engineers Press, Inc: New York.

[34] Chang, Y.H., and Yeh, C.H. (2004), A new air line safety index, Transportation Research Part B., 38, 369-383.

[35] Kahraman, C., Cebeci, U., and Ruan, D. (2004), Multi-attribute comparison of catering service companies using fuzzy AHP the case of Turkey, International Journal of Production Economics, 87, 171-184.

[36] Klir, G.J., and Yuan, B. (1995), Fuzzy Sets And Fuzzy Logic Theory and Application USA, Pearson Education: Inc. Publishing as Prentice Hall.
[37]Negi, D.S. (1989), Fuzzy analysis and optimization. Ph D thesis Department of Industrial Engineering Kansas state University.

[38] Ban, O.I. (2011), Fuzzy multicriteria decision making method applied to selection of the best touristic destinations, International journal of mathematical models and methods in applied sciences, $5(2)$.

[39] Buckley, J.J. (1985), Fuzzy hierarchical analysis, Fuzzy Sets and Systems, 17, 233247.

[40] Oladeinde, M.H., and Oladeinde, C.A. (2014), Metric distance ranking technique for fuzzy critical path analysis, J.Appl. Sci. Environ. Manage, 18(4), 583-589.

[41] Yang, M.S., and Ko, C.H. (1996), On a class of fuzzy c-numbers clustering procedures for fuzzy data, Fuzzy Sets and Systems, 84, 49-60.

[42] Md Saad, R., Ahmad, M.Z., and Abu, M.S. (2014), Hamming Distance Method with Subjective and Objective Weights for Personnel Selection, The Scientific World Journal, Article ID-865495. 